\title{
PRE-FACTIBILIDAD TÉCNICA EN LA GENERACIÓN DE ENERGÍA EÓLICA PARA PLANTAS CONVENCIONALES DE POTABILIZACIÓN DE AGUA: UN CASO REGIONAL COLOMBIANO
}

\section{TECHNICAL PRE-FEASIBILITY IN THE WIND ENERGY GENERATION FOR CONVENTIONAL WATER PURIFICATION PLANTS: A COLOMBIAN REGIONAL CASE}

\begin{abstract}
Carlos Alfonso Zafra ${ }^{1}$, Luis Hernando Castillo ${ }^{2}$, Santiago Andrés Rico ${ }^{3}$
${ }^{1}$ Ph.D. Ingeniería Ambiental, Profesor Asociado, Grupo de Investigación GIIAUD, Ingeniería Ambiental, Facultad del Medio Ambiente y Recursos Naturales, Universidad Distrital Francisco José de Caldas, Avda. Circunvalar Venado de Oro, Bogotá D.C., Colombia. Email: czafra@udistrital.edu.co; ${ }^{2}$ Ingeniero Ambiental, Grupo de Investigación GIIAUD, Ingeniería Ambiental, Universidad Distrital Francisco José de Caldas, Avda. Circunvalar Venado de Oro, Bogotá D.C., Colombia. Email: luiscastillo_m@ingenieros.com; ${ }^{3}$ Ingeniero Ambiental, Grupo de Investigación GIIAUD, Ingeniería Ambiental, Universidad Distrital Francisco José de Caldas, Avda. Circunvalar Venado de Oro, Bogotá D.C., Colombia. Email: santiagorico@ingenieros.com
\end{abstract}

Rev. U.D.C.A Act. \& Div. Cient. 16(1): 223 - 233, 2013

\section{RESUMEN}

Se presenta una metodología para analizar la pre-factibilidad técnica del aprovechamiento de la energía eólica, para el suministro eléctrico en plantas potabilizadoras de agua (PTAP), a través del tratamiento estadístico de registros climatológicos, el cálculo de la oferta energética y el dimensionamiento del aerogenerador. Adicionalmente, se muestra un nomograma para el cálculo de las anteriores variables, a partir de los registros de seis PTAP de la Sabana de Bogotá (Colombia): Principal, El Sosiego, Sibaté, Pablo VI, El Dorado y Yomasa. Las dos últimas PTAP, se tomaron como casos principales de estudio. Los resultados mostraron, para las PTAP El Dorado y Yomasa, a partir de sus caudales $(337,2$ y $12,1 \mathrm{~L} / \mathrm{s})$ y consumos energéticos máximos (37.322 y $3.571 \mathrm{kWh}$ ), que las condiciones climatológicas presentes a las alturas de diseño (50 y 10m) ofertaron una potencia eólica específica de 245,3 y $67,6 \mathrm{~W} / \mathrm{m}^{2}$, respectivamente. Por otro lado, se observó la existencia de una relación lineal entre el caudal tratado y la energía consumida por las seis PTAP en estudio $\left(R^{2}=0,99\right)$. Finalmente, al aplicar el nomograma de diseño eólico a las PTAP El Dorado y Yomasa, se evidenció un error medio de $0,54 \%$ y $1,25 \%$ en el cálculo de la energía consumida y la potencia eólica, respectivamente.

Palabras clave: Planta de tratamiento, potabilización de agua, caudal, energía eólica, aerogenerador.

\section{SUMMARY}

A methodology to analyze the technical pre-feasibility of the use of wind energy for electricity supply in water purification plants (WPP), through the statistical treatment of weather records, the calculation of the energy supply and the sizing of the wind turbine is presented. Additionally, a nomogram for calculation of the above variables from the records of six WPP of the Sabana de Bogotá (Colombia): Principal, El Sosiego, Sibaté, Pablo VI, El Dorado and Yomasa is exhibited. The last two WPP were taken as main study cases. The results showed for the WPP El Dorado and Yomasa from its maximum flow $(337.2$ y $12.1 \mathrm{~L} / \mathrm{s})$ and energy consumption $(37,322$ and $3,571 \mathrm{kWh})$ that the weather conditions present at the heights of design (50 and $10 \mathrm{~m}$ ) offer a specific wind power of 245.3 and $67.6 \mathrm{~W} / \mathrm{m}^{2}$, respectively. On the other hand, the existence of a linear relationship between the flow treated and the energy consumed by the six WPP in study $\left(R^{2}\right.$ $=0.99$ ) was noted Finally, the application of wind design nomogram to WPP El Dorado and Yomasa presented an average error of $0.54 \%$ and $1.25 \%$ in the calculation of the energy consumed and wind power, respectively.

Key words: Treatment plant, water purification, flow rate, wind energy, wind turbine. 


\section{INTRODUCCIÓN}

La escasez y los inconvenientes en la adquisición de información eólica en Colombia, los altos consumos energéticos y las consecuencias ambientales de la producción energética hacen necesario desarrollar investigaciones, que tengan por objeto evaluar el potencial del recurso para determinadas aplicaciones, asociadas con los sistemas de saneamiento básico. Por otro lado, agua y energía son dos de los temas más importantes en las agendas de desarrollo del entorno nacional e internacional. Asimismo, estos recursos se encuentran recíprocamente ligados: la producción de energía requiere de grandes volúmenes de agua, mientras que la potabilización y la distribución del agua es igualmente dependiente del fácil acceso y bajo costo de la energía (Mathioulakis et al. 2007).

A nivel mundial, la energía eólica cuenta con una capacidad instalada, que alcanzó 254.000MW (WWEA, 2012); por ejemplo, en España, esta energía cubre el 16\% de la demanda nacional de energía eléctrica (Espejo \& García, 2012). Por otro lado, Kalogirou (2005) argumentó que la energía eólica mundial podría proporcionar una cantidad de energía eléctrica igual a la demanda mundial de electricidad. En el caso específico de las plantas de potabilización de agua (PTAP), los consumos de energía eléctrica son elevados, principalmente, en lo relacionado con el control de los procesos de tratamiento (Qingfen \& Hui, 2011), implicando mayores costos, que son asumidos por las empresas prestadoras del servicio de agua potable y, por lo tanto, por los beneficiarios del sistema (Eltawil et al. 2009).

Como ejemplo de lo anterior, en España la PTAP de desalación de agua marina de Blanes en Girona posee un elevado consumo energético $(90.000 \mathrm{kWh})$, para tratar un caudal medio de 203L/s (Mujeriego, 2005). Igualmente, la PTAP Eguillor en Navarra, que requiere de un consumo promedio de $39.011 \mathrm{kWh}$, para tratar un caudal de 502L/s (MCP, 2009). El caso colombiano no es la excepción; por ejemplo, en la Sabana de Bogotá, la PTAP El Dorado, operada por la Empresa de Acueducto y Alcantarillado de Bogotá (E.A.A.B.), posee un elevado consumo de energía (33.158kWh), para tratar un caudal promedio de 301L/s (DAPD, 2004); asimismo, la PTAP "Principal" del municipio de Madrid (Cundinamarca), que posee un consumo energético de $11.500 \mathrm{kWh}$, para tratar un caudal promedio de 61,1L/s.

A partir de la perspectiva anterior, es preciso adelantar investigaciones que pretendan desarrollar metodologías para evaluar, desde el punto de vista técnico, la capacidad eólica de las áreas de influencia de las PTAP, para reducir los costos de tratamiento del agua y generar proyectos de producción más limpia. El desarrollo de este tipo de proyectos implica el conocimiento de la capacidad eólico-energética en las posi- bles zonas de actuación; sin embargo, el escaso número de estaciones climatológicas, la interrupción de las lecturas en los registros, los fallos en los instrumentos de medida, los errores asociados a cambios en las condiciones de medición y los errores de trascripción hacen necesaria la definición de metodologías adecuadas para la obtención de series continuas de datos climatológicos (Barrera et al. 2006).

Se han generado diversos métodos para la realización de estudios eólicos a partir del analisis de los registros existentes, como es el caso de la distribución de Weibull, basada en el análisis de los registros horarios de la velocidad del viento (Amar \& Elamouri, 2011; Fyrippis et al. 2010); los modelos de regresión lineal, apoyados en la obtención de registros diarios y mensuales de la velocidad del viento (Tavares et al. 2011); los estudios fundamentados en el análisis de perfiles logarítmicos del viento, donde los registros son recolectados de torres anemométricas, para determinar la rapidez a diversas alturas (Roballo \& Fisch, 2008) y los métodos de análisis tendencial, como lo son los modelos autorregresivos, integrados y de promedios móviles (ARIMA), que hacen una estimación predictiva en la generación de registros históricos (Kavasseri \& Seetharaman, 2009).

En los últimos años, varias PTAP, a nivel mundial, han optado por la energía eólica como fuente energética auto sostenible (Koschikowski \& Heijman, 2008; Park et al. 2011; Dehmas et al. 2011); por ejemplo, en España, las PTAP que se localizan cerca al mar e incorporan procesos de desalinización frecuentemente utilizan el potencial eólico, para satisfacer sus necesidades energéticas (Palomar \& Losada, 2011). Por otra parte, en países latinoamericanos, como México, se evalúa la posibilidad de utilizar este tipo de energía, para realizar el tratamiento del agua. El objetivo es reducir los costos asociados con la generación de energía termo-eléctrica y, por lo tanto, los costos de la potabilización del agua (Huacuz, 2005).

El objetivo principal del presente trabajo fue formular una metodología para el análisis de pre-factibilidad técnica en la generación de energía eólica para plantas convencionales de potabilización de agua (PTAP). Se pretendió identificar las variables climatológicas que afectan el cálculo de la potencia eólica específica y la relación entre el caudal tratado por la PTAP y el dimensionamiento preliminar de aerogeneradores. Adicionalmente, se desarrolló un nomograma para el dimensionamiento preliminar del aerogenerador a partir del caudal tratado por la PTAP.

\section{MATERIALES Y MÉTODOS}

Descripción del área de investigación: Las PTAP utilizadas, como casos principales de estudio, El Dorado y Yomasa, hacen parte del sistema de abastecimiento sur, de la ciudad de 
Bogotá D.C. (Colombia) y son operadas por la Empresa de Acueducto y Alcantarillado de Bogotá (E.A.A.B.) (Figura 1) y tienen una capacidad máxima de tratamiento de diseño de 1.600 y $40 \mathrm{~L} / \mathrm{s}$, respectivamente; sin embargo, actualmente, sus condiciones de operación alcanzan un caudal máximo de 337,2 y $12,1 \mathrm{~L} / \mathrm{s}$, respectivamente, es decir, por lectura directa en la PTAP.

La PTAP El Dorado, se encuentra localizada en la zona rural de Bogotá D.C., en el barrio Uval (Localidad de Usme), con un uso de suelo no urbano urbanizable y abastece a más de 300.000 habitantes (DAPD, 2004). Dentro de las instalaciones de la PTAP, se realizan procesos de aireación, de coagulación, de floculación, de sedimentación, de filtración, de desinfección y de estabilización de pH (DAPD, 2004), es decir, corresponde a un sistema convencional de potabilización. Por otro lado, la PTAP Yomasa, se encuentra en el límite de la zona urbana de Bogotá D.C., en la localidad de Usme, con un uso de suelo urbano no urbanizable y abastece a cerca de 10.000 habitantes (DAPD, 2004). Igualmente, sus procesos de tratamiento corresponden a un sistema convencional de potabilización.

El desarrollo eólico de la investigación, se fundamentó en los registros históricos durante ocho años de velocidad del viento y de temperatura, a 10 y $2 \mathrm{~m}$ sobre la superficie (i.e., diarios), respectivamente y registros mensuales de dirección del viento, a 10m sobre la superficie de siete estaciones

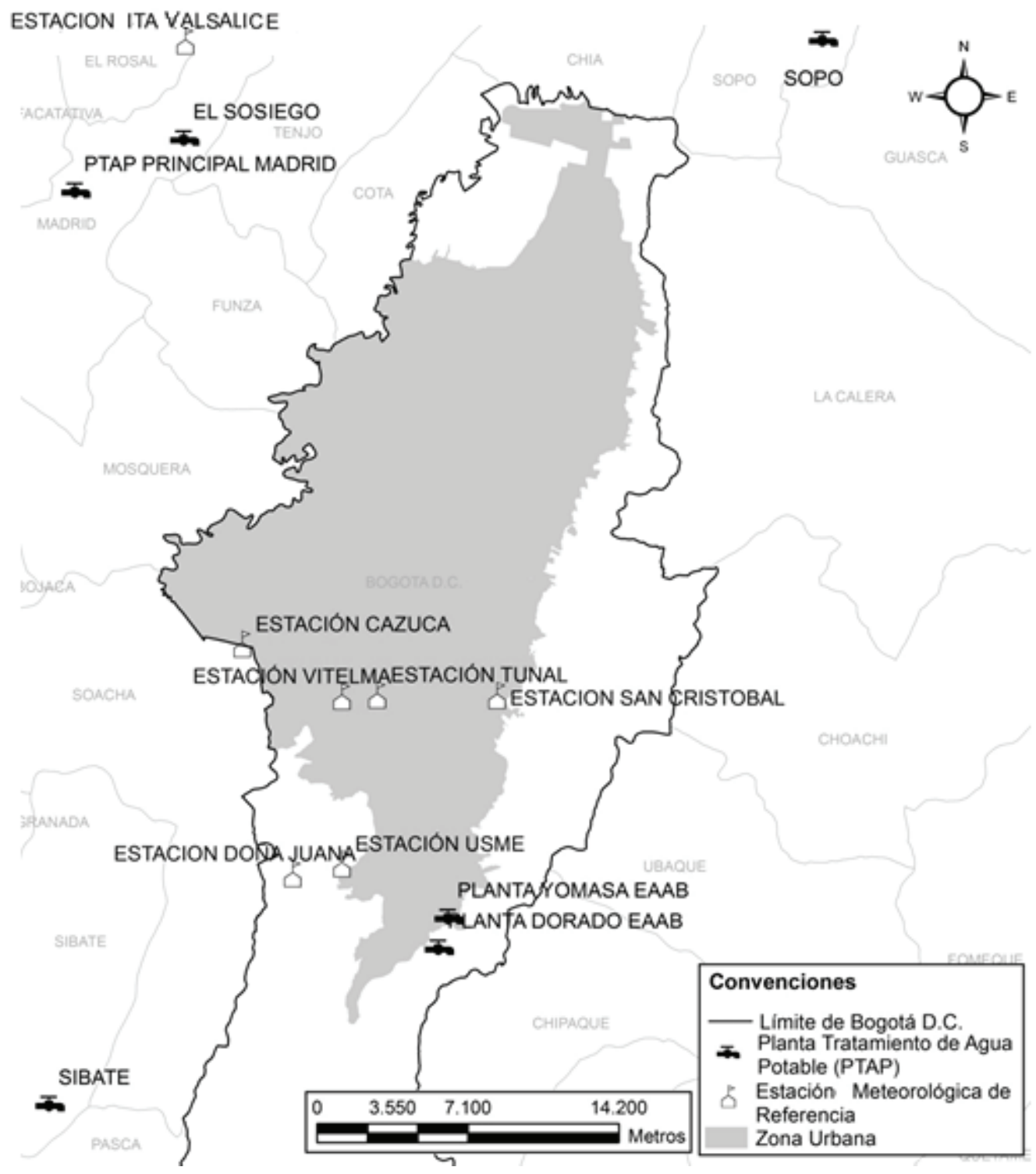

Figura 1. Localización de las PTAP y las estaciones climatológicas utilizadas para el desarrollo metodológico. 
meteorológicas de referencia: San Cristóbal, Tunal, Usme, Vitelma, Doña Juana, Cazucá e Ita Valsalice (Figura 1). De igual manera, se recopilaron los registros máximos y medios mensuales durante ocho años del caudal tratado y el consumo energético de seis PTAP, localizadas en la sabana de Bogotá, para el desarrollo del nomograma de diseño eólico
(Tabla 1 y Figura 1). A continuación, se presenta el desarrollo de la metodología propuesta (Figura 2) y el nomograma para el análisis de pre-factibilidad técnica en la generación de energía eólica para PTAP, con sistemas convencionales de tratamiento.

Tabla 1. PTAP utilizadas para el desarrollo metodológico.

\begin{tabular}{|c|c|c|c|c|c|c|c|c|c|}
\hline \multirow{2}{*}{$\begin{array}{c}\text { Caudal medio } \\
(\mathrm{L} / \mathbf{s})\end{array}$} & $\begin{array}{c}\text { Consumos de en- } \\
\text { ergía (kWh) }\end{array}$ & \multirow{2}{*}{ PTAP } & \multicolumn{7}{|c|}{ Proceso $^{\mathbf{a}}$} \\
\cline { 5 - 11 } & & & $\mathbf{1}$ & $\mathbf{2}$ & $\mathbf{3}$ & $\mathbf{4}$ & $\mathbf{5}$ & $\mathbf{6}$ & $\mathbf{7}$ \\
\hline 61,1 & 11.500 & Principal & & $\mathrm{X}$ & $\mathrm{X}$ & $\mathrm{X}$ & $\mathrm{X}$ & $\mathrm{X}$ & \\
\hline 10,1 & 2.220 & Sosiego & & $\mathrm{X}$ & $\mathrm{X}$ & $\mathrm{X}$ & $\mathrm{X}$ & $\mathrm{X}$ & \\
\hline 301 & 33.158 & El Dorado & $\mathrm{X}$ & $\mathrm{X}$ & $\mathrm{X}$ & $\mathrm{X}$ & $\mathrm{X}$ & $\mathrm{X}$ & $\mathrm{X}$ \\
\hline 9,2 & 2.511 & Yomasa & $\mathrm{X}$ & $\mathrm{X}$ & $\mathrm{X}$ & $\mathrm{X}$ & $\mathrm{X}$ & $\mathrm{X}$ & $\mathrm{X}$ \\
\hline 8,2 & 2.825 & Pablo VI & $\mathrm{X}$ & $\mathrm{X}$ & $\mathrm{X}$ & $\mathrm{X}$ & $\mathrm{X}$ & $\mathrm{X}$ & \\
\hline 25,9 & 4.735 & Sibaté ESP & & $\mathrm{X}$ & $\mathrm{X}$ & $\mathrm{X}$ & $\mathrm{X}$ & $\mathrm{X}$ & \\
\hline
\end{tabular}

a: 1: aireación; 2: coagulación; 3: floculación; 4: sedimentación; 5: filtración; 6: desinfección; 7: estabilización de pH

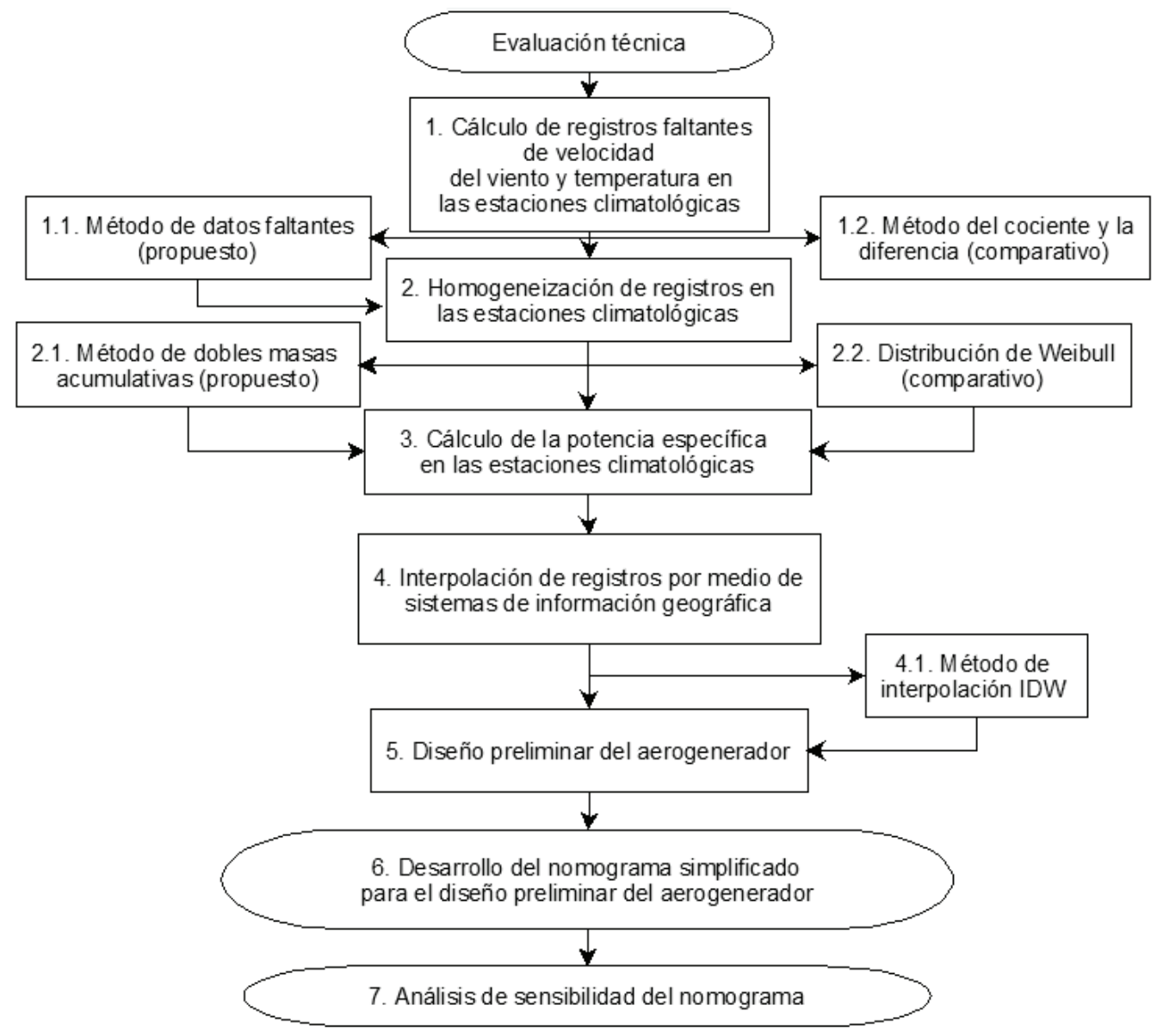

Figura 2. Estructura general de la metodología propuesta. 
Desarrollo metodológico: Para el cálculo de la potencia eólica específica, es decir, para los registros discontinuos y heterogéneos de velocidad del viento y temperatura de las estaciones climatológicas, se procedió a eliminar los datos extremos, basados en el criterio de la desviación estándar: se eliminaron los valores que se encontraron fuera del rango de la media, más o menos dos veces la desviación estándar (Holder, 1985). Posteriormente, la información diaria obtenida se agregó en promedios mensuales. Los registros faltantes se completaron, a partir del método de regresión múltiple entre estaciones y se utilizó el método de dobles masas acumulativas, para verificar la homogeneidad de los datos utilizados (Holder, 1985).

Los registros mensuales resultantes de velocidad y los obtenidos de la dirección del viento de las estaciones climatológicas (i.e., serie de datos), se analizaron a través de sus componentes vectorial y escalar aplicando el programa WRPLOT, diseñado por la Agencia de Protección Ambiental de EE.UU. (i.e., diseño de rosa de vientos); a partir de lo anterior, se obtuvo la dirección predominante del viento (Cheremisinoff, 2002; Lima et al. 2012). Por otro lado, se obtuvieron los perfiles logarítmicos de velocidad del viento con los registros mensuales sobre cada estación climatológica y para diferentes alturas, con respecto de la superficie del suelo: 10, 20, 30, 40 y 50m, a partir de lo expuesto por Tavares et al. (2011). En este sentido, los valores de rugosidad fueron los correspondientes a terrenos abiertos con pastoreo intensivo $(\mathrm{Zo}=0,03)$ y cerrados de centros poblados $(\mathrm{Zo}=$ 1) (IDEAM, 2006). Finalmente, se realizaron correcciones de los perfiles logarítmicos del viento, por medio del factor de potencia eólica $K_{E}$ (Ecuación 1); este factor disminuyó las fluctuaciones generadas en la estimación del potencial eólico específico. Lo anterior, debido a que la potencia dependió del cubo de la velocidad haciéndola sensible a cambios en la rapidez del viento.

$$
\mathrm{k}_{\mathrm{E}}=\frac{\frac{1}{\mathrm{~N}} \sum_{\mathrm{n}=1}^{\mathrm{N}} \mathrm{V}_{\mathrm{n}}^{3}}{\left[\frac{1}{\mathrm{~N}} \sum_{\mathrm{n}=1}^{\mathrm{N}} \mathrm{V}_{\mathrm{n}}\right]^{3}}
$$

(Ecuación 1) (Lysen, 1983)

Donde $\mathrm{k}_{\mathrm{E}}$, representó el factor de potencia eólica, adimensional; $\mathrm{N}$, la cantidad total de meses presentes en la serie de datos y $\mathrm{Vn}$, la velocidad media mensual en $\mathrm{m} / \mathrm{s}$.

Por otra parte, se empleó la expresión del gradiente térmico vertical (Ecuación 2), para determinar la temperatura a diferentes alturas con respecto de la superficie del suelo: 10,20 , 30,40 y $50 \mathrm{~m}$.

$$
\mathrm{T}_{\mathrm{Z}}=\mathrm{T}_{\mathrm{zo}}-\frac{0,65}{100} \cdot \mathrm{Z}
$$

(Ecuación 2)

(García \& Castejón, 1986)

Donde $\mathrm{Tz}$, representó la temperatura promedio mensual a una altura determinada en ${ }^{\circ} \mathrm{C}$; Tzo, la temperatura promedio mensual a la altura de referencia en ${ }^{\circ} \mathrm{C}$ (i.e., a $2 \mathrm{~m}$ ) y Z, la diferencia de altura en metros. A partir de lo anterior, se calculó la tensión de vapor del aire "e", como una función de la temperatura (Ecuación 3); variable utilizada para la corrección de la densidad del aire.

$$
\mathrm{e}=\exp \left[-\frac{6763,6}{\mathrm{~T}}\right]-4,9283 \cdot \ln (\mathrm{T})+54,23 \quad \begin{array}{r}
\text { (Ecuación 3) } \\
\text { (Sozzi et al. 1998) }
\end{array}
$$

Posteriormente, se determinó la presión atmosférica para las diferentes alturas en evaluación (Ecuación 4):

$$
\mathrm{P}_{\mathrm{S}}=\mathrm{P}_{00} \cdot\left(\frac{\mathrm{P}_{00}}{850}\right)^{-\frac{2}{\mathrm{H}_{850}}} \quad \text { (Ecuación 4) (Dudhia et al. 2005) }
$$

Donde $\mathrm{P}_{\mathrm{s}}$, representó la presión atmosférica en $\mathrm{hPa} ; \mathrm{P}_{\mathrm{oo}}$, la presión atmosférica a nivel del mar en $\mathrm{hPa} ; \mathrm{H}_{850}$, la altura geopotencial a $850 \mathrm{hPa}$ de presión (i.e., 1480 m.s.n.m.) y z, la altura geopotencial con respecto al nivel medio del mar en metros (i.e., a condiciones locales). Seguidamente, se cálculo la densidad del aire teniendo en cuenta un ajuste a la constante universal de los gases ideales (Ecuación 5), a partir de la tensión de vapor del aire (Dudhia et al. 2005).

$$
\mathrm{R}^{*}=\mathrm{R} \cdot\left(1+\frac{3}{8} \cdot \frac{\mathrm{e}}{\mathrm{Ps}}\right) \quad(\text { Ecuación 5) (García \& Castejón, 1986) }
$$

Donde $\mathrm{R}^{*}$, representó la constante universal de los gases ideales en $\mathrm{J} / \mathrm{kg}^{\circ} \mathrm{K}$; $\mathrm{R}$, la constante universal de los gases ideales: $286 \mathrm{~J} / \mathrm{kg}^{\circ} \mathrm{K}$; y e y Ps, la tensión de vapor y la presión atmosférica en $\mathrm{Pa}$, respectivamente. A partir de lo anterior, se determinó la densidad del aire mensual en $\mathrm{kg} / \mathrm{m}^{3}\left(\boldsymbol{\rho}_{\mathrm{h}}\right)$, para las diferentes alturas en evaluación (Ecuación 6):

$$
\rho_{\mathrm{h}}=\frac{\mathrm{P}_{\mathrm{s}}}{\mathrm{R} \cdot \mathrm{T}_{\mathrm{z}}} \quad \text { (Ecuación 6) (García \& Castejón, 1986) }
$$

Posteriormente, se determinó la densidad de potencia o el potencial eólico específico, a partir de los registros mensuales de densidad del aire y velocidad del viento, obtenido en cada una de las estaciones climatológicas y para las diferentes alturas de investigación (Ecuación 7):

$$
\left(\frac{P_{\mathrm{e}}}{\mathrm{A}}\right)=\frac{1}{2} \cdot \mathrm{k}_{\mathrm{E}} \cdot \rho_{\mathrm{h}} \cdot \overline{\mathrm{V}}^{3} \quad \text { (Ecuación 7) (Lysen, 1983) }
$$

Donde el término $P_{e} / A$, representó la densidad de potencia o potencia eólica específica en $\mathrm{W} / \mathrm{m}^{2} ; \mathrm{k}_{\mathrm{E}}$, el factor de corrección de la potencia eólica específica, adimensional; $\rho_{\mathrm{h}}$, la densidad del aire en $\mathrm{kg} / \mathrm{m}^{3}$; V, la velocidad del viento en $\mathrm{m} / \mathrm{s}$; $\mathrm{P}_{\mathrm{e}}$, la potencia eólica en $\mathrm{W}$ y $\mathrm{A}$, el área de barrido perpendicular a la dirección del viento en $\mathrm{m}^{2}$.

A partir de los resultados obtenidos en cada una de las estaciones climatológicas de referencia, se interpolaron con el 
programa ArcGIS los registros de potencia eólica específica, para obtener las magnitudes correspondientes en cada PTAP de estudio (i.e., método del inverso de las distancias al cuadrado, IDW). El método IDW asumió que cada punto de interés (i.e., PTAP en estudio) tuvo una influencia en su vecindad que disminuyó con la distancia (González \& Bosque, 2008), es decir, el método dio más peso a los puntos más cercanos a la PTAP en estudio, que aquellos que estuvieron más alejados.

Diseño preliminar del aerogenerador: El diseño del aerogenerador, se fundamentó en la determinación del área de barrido (Ecuación 7). Como se pudo observar, el área de barrido del aerogenerador, se determinó como la relación entre la potencia eólica $\left(\mathrm{P}_{\mathrm{e}}\right.$; en $\left.\mathrm{W}\right)$ y la densidad de potencia $\left(\mathrm{P}_{\mathrm{e}} / \mathrm{A}\right.$; en $\mathrm{W} / \mathrm{m}^{2}$ ). Adicionalmente, en el cálculo del área de barrido, se incluyó la conversión de la potencia eólica a potencia eléctrica, teniendo en cuenta las pérdidas por conversión mecánica, Ley de Betz (Solano, 2008) y las pérdidas por conversión eléctrica (Le Gourières, 1982) (Ecuación 8):

$$
P_{\text {eléctrica }}=\eta \cdot P_{\text {mecánica }}=\eta \cdot C_{P} \cdot P_{e}
$$

(Ecuación 8)

(Hernández, 2005)

Donde $\mathrm{P}_{\text {eléctrica, }}$ representó la potencia eléctrica en $\mathrm{kW} ; \mathrm{P}_{\text {mecá- }}$ nica, la potencia mecánica en $\mathrm{kW} ; \eta$, la eficiencia de los equipos transformadores de energía eléctrica, equivalente a 0,85 (i.e., por registro en la PTAP); $\mathrm{C}_{\mathrm{p}}$, representó el límite de Betz, equivalente a 0,59 y $\mathrm{P}_{\mathrm{e}}$, la potencia eólica en $\mathrm{kW}$.

Las prestaciones energéticas de cada PTAP, se definieron a partir de sus consumos mensuales de energía (i.e., $\mathrm{P}_{\text {eléctrica; }}$ Ecuación 8). La potencia eléctrica instalada en cada PTAP correspondió al valor máximo mensual de consumo energético. A partir de lo anterior, se determinó la potencia eléctrica de diseño con respecto al número de aerogeneradores implementados.

Se realizó el cálculo del número de palas del aerogenerador, donde $\lambda$, correspondió al cociente entre la velocidad de punta de la pala (i.e., velocidad tangencial en el movimiento circular uniforme de las palas; Ecuación 9) y la velocidad del viento.

$\mathrm{Vpp}=(\mathrm{w} \cdot \pi \cdot \varnothing) / 60$

(Ecuación 9) (Hernández, 2005)

Donde $\mathrm{V}_{\mathrm{pp}}$, representó la velocidad de punta de la pala en $\mathrm{m} / \mathrm{s}$ y $\boldsymbol{\omega}$, la velocidad angular en revoluciones por minuto (RPM) del alternador seleccionado, obtenida, en este caso, del catálogo del fabricante ALXION (2010). La relación entre $\lambda$ y el número de palas, se determinó, a partir de lo sugerido por Le Gourières (1982): para un valor de $\lambda$ de $1,2,3,4$, 5-8 y mayor de 8 , le correspondió un número de palas entre 6-20, 4-12, 3-8, 3-5, 2-4 y 1-2, respectivamente.
Por último, se asumió un coeficiente de simultaneidad de 1,0 , es decir, se asumió una oferta o potencia mínima de suministro de energía del aerogenerador, capaz de alimentar continuamente el sistema eléctrico, a su máxima capacidad (Le Gourières, 1982). Finalmente, para obtener la potencia eléctrica de diseño ( $P_{\text {eléctrica; }}$ Ecuación 8 ), se adicionó un $10 \%$ a la potencia eléctrica demandada por el sistema; este incremento, se debió a los requerimientos energéticos de las líneas de conducción eléctrica (Le Gourières, 1982).

Desarrollo del nomograma para el dimensionamiento preliminar del aerogenerador en PTAP: El nomograma de diseño preliminar del aerogenerador, se desarrolló en cinco cuadrantes, a partir de los datos obtenidos para las seis PTAP de investigación (Tabla 1 y Figura 3). Las curvas al interior del primer cuadrante, se obtuvieron a partir de la ecuación, para el cálculo de la potencia eólica específica (Ecuación 7). Se desarrollaron tres curvas dependiendo de la densidad del aire: $0,90,1,0$ y $1,23 \mathrm{~kg} / \mathrm{m}^{3}$ (Figura 3, cuadrante 1). Seguidamente, en el segundo cuadrante, se evaluó el área de barrido (i.e., diámetro), a partir del cálculo de la longitud de pala del aerogenerador (Ecuación 7). Se desarrollaron cinco curvas, para diámetros de barrido entre 8-25m (Figura 3, cuadrante 2).

Por otro lado, al evaluar la relación entre el caudal y la energía consumida para las seis PTAP de investigación, se observó que la tendencia lineal fue la que mejores resultados produjo bajo condiciones medias $\left(R^{2}=0,99\right)$ (Figura 3, cuadrante 3 ). El modelo lineal obtenido fue el siguiente:

$$
P C=104,06 \cdot Q+2277,7
$$

(Ecuación 10)

Donde PC, representó la energía consumida en kWh y Q, el caudal tratado por la PTAP en L/s. Finalmente, en el cuarto y quinto cuadrante, se realizó el cálculo del número de palas del aerogenerador; donde $\lambda$, correspondió el cociente entre la velocidad de punta de la pala y la velocidad del viento (Ecuación 9).

Como se puede observar, el nomograma de diseño fue desarrollado con dos parámetros de entrada para su aplicación: (i) velocidad del viento (Figura 3, cuadrante 1) y (ii) caudal tratado por la PTAP (Figura 3, cuadrante 3). Adicionalmente, se incluyeron al interior del nomograma dos líneas guía, para facilitar su aplicación, a partir de los dos parámetros de entrada mencionados anteriormente.

\section{RESULTADOS Y DISCUSIÓN}

Ejemplo de aplicación de la metodología propuesta: PTAP El Dorado y Yomasa: El potencial eólico específico o densidad de potencia, se determinó a partir de los registros de velocidad del viento, es decir, continuos y homogéneos (Ecua- 


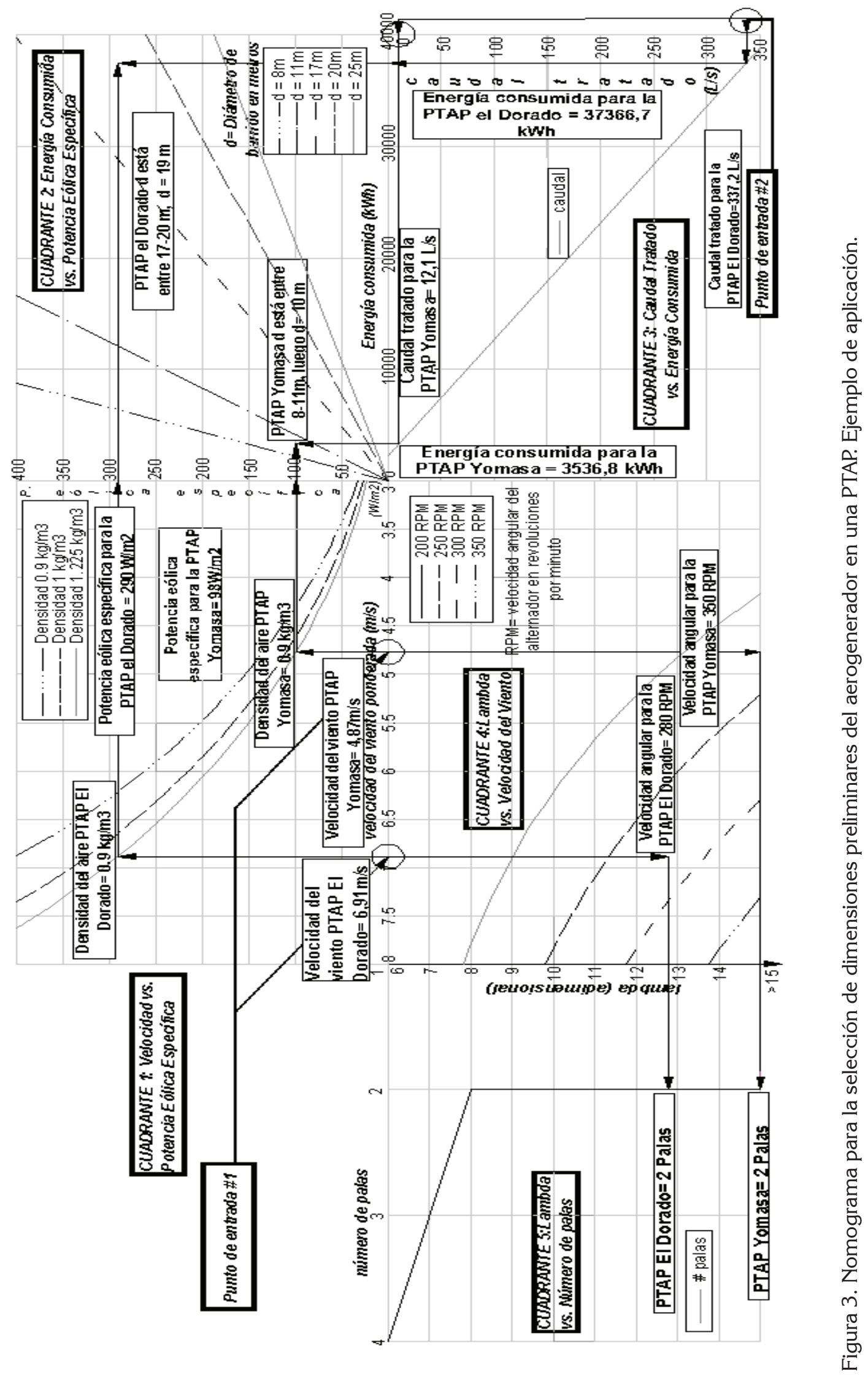


ción 7 y Tabla 2). Como es sabido, los registros de presión atmosférica, de temperatura y de tensión de vapor para la determinación de la densidad de potencia fueron corregidas por elevación y por variaciones climáticas (ecuaciones 2, 3, 4,5 y 6).

Diseño preliminar del aerogenerador: En el diseño preliminar del sistema eólico para cada PTAP, se consideró el uso de dos aerogeneradores, en otras palabras, se tuvo en cuenta probables labores de mantenimiento y alternancia en el uso durante su operación. La altura de diseño de los aerogeneradores, se determinó a partir de los consumos energéticos máximos de las PTAP El Dorado (37.322 kWh) y Yomasa (3.571 kWh) (Tabla 2).

Igualmente, se consideraron principios de proporcionalidad de la estructura eólica: la relación entre la longitud de la pala y la altura de la torre (Hernández, 2005). Así que la altura de diseño determinada para los aerogeneradores en las PTAP El Dorado y Yomasa fue de 50 y 10 m, respectivamente (Tabla 2). La determinación del área de barrido incluyó la conversión de la potencia eólica a potencia eléctrica teniendo en cuenta las perdidas por conversión mecánica, Ley de Betz (Solano, 2008) y las pérdidas por conversión eléctrica (Le

Tabla 2. Parámetros de diseño para el sistema eólico de las PTAP El Dorado y Yomasa.

\begin{tabular}{|c|c|c|}
\hline Parámetro & $\begin{array}{l}\text { PTAP } \\
\text { El Dorado }\end{array}$ & $\begin{array}{l}\text { PTAP } \\
\text { Yomasa }\end{array}$ \\
\hline \multicolumn{3}{|l|}{ Prestaciones energéticas } \\
\hline Energía máxima requerida $(\mathrm{kWh})$ & 37.322 & 3.571 \\
\hline Número de aerogeneradores & 2 & 2 \\
\hline $\begin{array}{l}\text { Energía requerida para el diseño del aerogenera- } \\
\text { dor }(\mathrm{kWh})\end{array}$ & 18.661 & 1.786 \\
\hline Potencia eléctrica demandada $(\mathrm{kW})$ & 25,9 & 2,5 \\
\hline Factor de uso simultaneo & 1 & 1 \\
\hline Energía mínima (kWh) & 18.661 & 1.786 \\
\hline \multicolumn{3}{|l|}{ Diseño eólico } \\
\hline Altura de diseño $(m)$ & 50 & 10 \\
\hline Dirección predominante del viento & Sur & Sur \\
\hline Velocidad del viento $(\mathrm{m} / \mathrm{s})$ & 6,9 & 4,9 \\
\hline Densidad del aire $\left(\mathrm{kg} / \mathrm{m}^{3}\right)$ & 0,9 & 0,9 \\
\hline Potencia eólica específica $\left(\mathrm{W} / \mathrm{m}^{2}\right)$ & 245 & 68 \\
\hline \multicolumn{3}{|l|}{ Diseño del aerogenerador } \\
\hline Altura de diseño $(\mathrm{m})$ & 50 & 10 \\
\hline Diámetro de barrido $(\mathrm{m})$ & 17 & 10 \\
\hline Área del rotor $\left(\mathrm{m}^{2}\right)$ & 227 & 79 \\
\hline Área de barrido $\left(\mathrm{m}^{2}\right)$ & 454 & 157 \\
\hline Número de palas & 2 & 2 \\
\hline Tipo de alternador/velocidad angular (RPM) & $800 \mathrm{STK}^{2} \mathrm{M}^{\mathrm{a}} / 280$ & $800 \mathrm{STK} 4 \mathrm{M}^{\mathrm{a}} / 350$ \\
\hline Potencia eólica $(\mathrm{kW})$ & 56 & 5,3 \\
\hline Potencia mecánica $(\mathrm{kW})$ & 33 & 3,1 \\
\hline Potencia eléctrica $(\mathrm{kW})$ & 28 & 2,7 \\
\hline Potencia del aerogenerador $(\mathrm{kW})$ & 43 & 4,1 \\
\hline Caudal máximo de tratamiento (L/s) & 337,2 & 12,1 \\
\hline
\end{tabular}

a: alternadores marca ALXION 
Gourières, 1982). Finalmente, para los dos casos en estudio, los aerogeneradores requirieron de dos palas (Tabla 2).

Ejemplo de aplicación del nomograma para el dimensionamiento preliminar del aerogenerador en una PTAP: El nomograma fue aplicado a los dos casos principales de estudio: PTAP El Dorado y PTAP Yomasa. Como es sabido, el nomograma de diseño fue desarrollado con dos parámetros de entrada para su aplicación: (i) velocidad del viento (Figura 3 , cuadrante 1: velocidad versus potencia eólica específica) y (ii) caudal tratado por la PTAP (Figura 3, cuadrante 3: Caudal tratado versus energía consumida). Los datos de entrada para el uso del nomograma provinieron del análisis eólico de la zona de interés. Por lo tanto, la velocidad predominante del viento para las PTAP El Dorado y Yomasa fue de $6,91 \mathrm{~m} / \mathrm{s}$ (altura: $50 \mathrm{~m}$ ) y $4,87 \mathrm{~m} / \mathrm{s}$ (altura: $10 \mathrm{~m}$ ), respectivamente. Adicionalmente, como dato de partida, se determinó el caudal máximo tratado por cada PTAP, durante el mes de máximo consumo energético: 337,2L/s, para la PTAP El Dorado y 12,1L/s, para la PTAP Yomasa (Figura 3, cuadrante 3).

Los resultados obtenidos por la aplicación del nomograma fueron comparados con los del desarrollo metodológico propuesto, con respecto del máximo consumo energético de las PTAP El Dorado y Yomasa. Los resultados mostraron que al utilizar el nomograma de diseño eólico se presentaron diferencias para las PTAP El Dorado y Yomasa, de 0,12\% y $0,96 \%$, respectivamente, en el cálculo del consumo energético $(\mathrm{kWh})$ (Figura 3, cuadrante 3: caudal tratado versus energía consumida).

La anterior variación, probablemente se debió, a la diferencia en la tecnología empleada para el control de los procesos de tratamiento, es decir, en las PTAP de la Sabana de Bogotá, a medida que aumenta el caudal tratado, se incrementa la tecnología para el control de los procesos y, por lo tanto, se incrementa el consumo energético de la PTAP, generando un menor error en la estimación, a través de la aplicación del nomograma de diseño desarrollado.

Finalmente, al aplicar el nomograma de diseño, se generó un error de 2,49\% y $0 \%$ en el cálculo de la potencia eólica para la PTAP El Dorado $(57,1 \mathrm{~kW})$ y Yomasa $(5,3 \mathrm{~kW})$, respectivamente (Figura 3, cuadrante 2: Energía consumida versus potencia eólica específica). Como se puede observar, existió una relación positiva entre el error y la potencia eólica determinada para cada PTAP, es decir, a medida que aumenta el consumo energético y, por lo tanto, la potencia eólica requerida por la PTAP, aumenta el error en el pronóstico, mediante el empleo del nomograma de diseño desarrollado.

Los resultados muestran que la generación de energía eólica para las PTAP en estudio es técnicamente factible. El diseño eólico muestra para las PTAP El Dorado y Yomasa, a partir de sus máximos caudales $(337,2$ y $12,1 \mathrm{~L} / \mathrm{s})$ y consumos energéticos (37.322 y $3.571 \mathrm{kWh})$, que las condiciones climatológicas presentes a las alturas de diseño (50 y 10m) ofertaron una potencia eólica específica de 245,3 y 67,6W/ $\mathrm{m}^{2}$, respectivamente.

Por otro lado, se observa la existencia de una relación lineal entre el caudal tratado y la energía consumida por las seis PTAP en estudio de la Sabana de Bogotá $\left(R^{2}=0,99\right)$. La anterior relación permitirá realizar el pronóstico de la energía consumida para nuevos proyectos de potabilización de agua. Es importante resaltar, que las técnicas de tratamiento implementadas por las PTAP en estudio corresponden a sistemas convencionales de potabilización de agua. Adicionalmente, el uso del nomograma de diseño eólico en la PTAP El Dorado y Yomasa presenta un error medio de 0,54\% y 1,25\% en el cálculo de la energía consumida y la potencia eólica, respectivamente, en comparación con la metodología propuesta.

Finalmente, la metodología propuesta permite profundizar en el diseño de sistemas eólicos para una PTAP en nuestra región y puede ser utilizada como una guía de selección del equipamiento requerido para la generación de energía eléctrica con este recurso.

Agradecimientos: Los autores desean agradecer el apoyo brindado por la Empresa de Acueducto y Alcantarillado de Bogotá, la Secretaría Distrital de Ambiente de Bogotá, la Corporación Autónoma Regional de Cundinamarca, el Instituto de Hidrología, Meteorología y Estudios Ambientales, la Superintendencia de Servicios Públicos, la Empresa de Servicios Públicos de Sopó y la Empresa de Servicios Públicos Domiciliarios de Sibaté. Conflictos de intereses: El manuscrito fue preparado y revisado con la participación de todos los autores, quienes declaramos que no existe ningún conflicto de intereses que ponga en riesgo la validez de los resultados presentados. Financiación: Este estudio fue financiado por el Grupo de Investigación en Ingeniería Ambiental de la Universidad Distrital F.J.C (GIIAUD).

\section{BIBLIOGRAFÍA}

1. ALXION. 2010. Technical Characteristics STK Wind Turbine Alternators. Ed. ALXION (France). 1p. Disponible desde internet en: http://www.alxion.com/ products/stk-alternators (con acceso 02/11/2010).

2. AMAR, F.B.; ELAMOURI, M. 2011. A new theoretical model for modeling the wind speed frequency distribution (Tunisia). Renew. Energ. (USA). 1(4):306-313.

3. BARRERA, A.; LLASAT, M.C.; BARRIENTOS, M. 2006. Estimation of extreme flash flood evolution in Barce- 
lona County from 1351 to 2005. Nat. Hazards Earth Syst. Sci. (Germany). 6(4):505-518.

4. CHEREMISINOFF, N. 2002. Handbook of air pollution prevention and control. Ed. Butterworth-Heinemann (USA). 562p.

5. DAPD. 2004. Plan de ordenamiento territorial de la localidad de Usme. Ed. Departamento Administrativo de Planeación Distrital de Bogotá (Colombia). 103p.

6. DEHMAS, D.A.; KHERBA, N.; HACENE, F.B.; MERZOUK, N.K.; MERZOUK, M.; MAHMOUDI, H.; GOOSEN, M.F.A. 2011. On the use of wind energy to power reverse osmosis desalination plant: A case study from Ténès (Algeria). Renew Sust. Energ. Rev. (USA). 15(2):956-963.

7. DUDHIA, J.; GILL, D.; MANNING, K.; WANG, W.; BRUYERE, C. 2005. Mesoscale Modeling System Tutorial Class Notes and User Guide: MM5 Modeling System Version 3. Ed. National Center for Atmospherich Research (USA). 117p. Disponible desde internet en: http://www.mmm.ucar.edu/mm5/documents/tutorial-v3-notes.html (con acceso el 15/11/2010).

8. ELTAWIL, M.A.; ZHENGMING, Z.; YUAN, L.A. 2009. Review of renewable energy technologies integrated with desalination systems. Renew Sust. Energ. Rev. 13(9):2245-2262.

9. ESPEJO, C.; GARCÍA, R. 2012. La energía eólica en la producción de electricidad en España. Rev. Geogr. Norte Gd. (Chile). 51(1):115-136.

10. FYRIPPIS, I.; AXAOPOULOS, P.J.; PANAYIOTOU, G. 2010. Wind energy potential assessment in Naxos Island, Greece. Appl. Energ. (Sweden). 87(2):577-586.

11. GARCÍA, C.; CASTEJÓN, F. 1986. Problemas de meteorología: estática y termodinámica de la atmósfera. Ed. Instituto Nacional de Meteorología de España (España). 242p.

12. GONZÁLEZ, D.; BOSQUE, J. 2008. Generación de un mapa de vientos en un SIG. Bol. Asoc. Geóg. Españoles (España). 47(1):51-77.

13. HERNÁNDEZ, J. 2005. Diseño de un aerogenerador de baja potencia. Ed. Universidad Politécnica de Cataluña (España). 74p.

14. HOLDER, R.L. 1985. Multiple regression in Hydrology. Ed. Galliard Ltd. (United Kingdom). 143p.
15. HUACUZ, J.M. 2005. The road to green power in Mexicoreflections on the prospects for the large-scale and sustainable implementation of renewable energy. Energ. Policy (United Kingdom). 33(16):2087-2099.

16. IDEAM. 2006. Atlas de viento y energía eólica de Colombia. Ed. Instituto de Hidrología, Meteorología y Estudios Ambientales (Colombia). 169p.

17. KALOGIROU, S.A. 2005. Seawater desalination using renewable energy sources. Progr. Energy Combustion Sci. (USA). 31(3):242-281.

18. KAVASSERI, R.G.; SEETHARAMAN, K. 2009. Day-ahead wind speed forecasting using f-ARIMA models. Renew. Energ. 34(5):1388-1393.

19. KOSCHIKOWSKI, J.; HEIJMAN, B. 2008. Renewable energy drives desalination processes in remote or arid regions. Membr. Techn. (United Kingdom). 8:89.

20. LE GOURIÈRES, D. 1982. Energía eólica: Teoría, concepción y cálculo práctico de las Instalaciones. Ed. Masson S.A. (España). 284p.

21. LIMA, F.J.L.; CAVALCANTI, E.P.; POUZA, E.P.; SILVA, E.M. 2012. Evaluation of the wind power in the state of Paraíba using the mesoscale atmospheric model brazilian developments on the regional atmospheric modelling system (brams). Renew. Energ. In Press. Doi: $10.5402 / 2012 / 847356$.

22. LYSEN, E. 1983. Introduction to Wind Energy: Basic and Advanced Introduction to Wind Energy with Emphasis on Water Pumping Windmills. Ed. Consultancy Services Wind Energy Developing Countries (The Netherlands). 310p.

23. MATHIOULAKIS, E.; BELESSIOTIS, V.; DELYANNIS, E. 2007. Desalination by using alternative energy: Review and state of the art. Desalination (United Kingdom). 203(1-3):346-365.

24. MCP. 2009. Informe de gestión. Ed. Mancomunidad de la Comarca de Pamplona (España). 4p. Disponible desde internet en: http://www.mcp.es/memoria2009/themed/mcp10/files/pdfs/estaciones-detratamiento-de-agua-potable_es.pdf (con acceso el 02/11/2010).

25. MUJERIEGO, R. 2005. La reutilización planificada del agua para regadío. Ed. Universidad Politécnica de Cataluña (España). 20p. Disponible desde internet 
en: http://www.geoscopio.com/empresas/fenacore2/ XI\%20CONGRESO\%20PALMA/Ponencia\%20\%20 R\%20Mujeriego.pdf (con acceso el 02/11/2010).

26. PALOMAR, P.; LOSADA, I.J. 2011. Desalination in Spain: Recent developments and recommendations. Desalination. 255(1-3):97-106.

27. PARK, G.L.; SCHÄFER, A.I.; RICHARDS, B.S. 2011. The effect of wind speed fluctuations on the performance of a wind-powered membrane system for brackish water desalination. J. Membr. Sci. (USA). 370(12):34-44.

28. QINGFEN, M.A.; HUI, L. 2011. Wind energy technologies integrated with desalination systems: Review and state-of-the-art. Desalination (277(1-3):274-280.

29. ROBALLO, S.T.; FISCH, G. 2008. Escoamento atmosférico no Centro de Lançamento de Alcântara (CLA): parte I - aspectos observacionais. Rev. Bras. Meteorol. 23(4):501-509.

30. SOLANO, L.L. 2008. Análisis de un parque eólico: Influencia en la tensión de la regulación de la potencia reactiva de sus aerogeneradores. Ed. Universidad Carlos III de Madrid (España). 183p.

31. SOZZI, R.; FAVARON, M.; GEORGIADIS, T. 1998. Method for the estimation of surface roughness and similarity function of the wind speed vertical profile. J. Appl. Meteorol. (USA). 37(5):461-469.

32. TAVARES, M.A.; DA SILVA, E.M.; BRAVO, J.M. 2011. Estimativa dos recursos eólicos no litoral cearense usando a teoria da regressão linear. Rev. Bras. Meteorol. 26(3):349-366.

33. WORLD WIND ENERGY ASSOCIATION WWEA. 2012. 2012 half year report. Ed. The World Wind Energy Association (Germany). 8p. Disponible desde internet en: http://www.wwindea.org/webimages/Halfyear_report_2012.pdf (con acceso 29/10/2012).

Recibido: Mayo 4 de 2012

Aceptado: Enero 22 de 2013 